\title{
New Year's resolution: increase the impact factor of this journal
}

\author{
Nagara Tamaki ${ }^{1}$
}

(C) Japan Radiological Society 2015

Thanks to the continuing support of the editorial board members, expert reviewers, and the editorial team from Springer, the Japanese Journal of Radiology (JJR) is making good progress at the start of 2016. This journal received nearly 600 manuscript submissions in 2015 , and the number of submissions has increased by $20-30 \%$ each year. Approximately two-thirds of those manuscripts and half of the articles accepted came from countries outside Japan, which suggests that JJR is now a truly international journal.

Given the significant increase in the manuscript submission rate to our journal, now would seem to be a good time to consider how to improve journal quality. Over the last 20 years, the journal impact factor (JIF) has assumed increasing importance among authors, academic centers, and publishers as a means to gauge the quality of the scientific work published by a particular journal. The JIF of a journal is calculated as the number of times that works (e.g., articles) published in that journal in the previous two years were cited in other indexed publications divided by the number of works published in the journal over the same period. Thus, in order to increase the JIF, the number of citations of works published in the journal must be increased and/or the number of works published in it during the two-year period should be reduced. Due to the high quality of the papers that have been published in our journal, the number of citations of those works has significantly increased. On the other hand, despite a low acceptance rate, the number of publications included in JJR has increased

Nagara Tamaki

natamaki@med.hokudai.ac.jp

1 Department of Nuclear Medicine, Hokkaido University Graduate School of Medicine, North 15, West 7, Kita-ku, Sapporo 060-8638, Japan each year. As a result, the JIF for this journal has increased moderately each year rather than dramatically-from 0.730 (in 2012) to 0.742 (in 2013) to 0.837 (in 2014).

We have discussed how to improve the JIF of the Japanese Journal of Radiology among the editorial board members. While many case reports have been submitted to the journal (indeed, almost half of all submissions to JJR are case reports, particularly outside from Japan), only a limited number of high-quality case reports have been selected for publication, based on their scientific and educational value $[1,2]$. However, those case reports have not been cited in other publications. Therefore, we have decided that JJR will no longer include case reports. Actually, several other journals with high JIFs have recently begun to exclude case reports, although you may also see some new journals that are willing to publish case reports. We highly recommend that you create other types of articles instead of case reports (e.g., by collecting more cases and/or adding important concepts about the cases) and submit those to JJR. High-quality original articles, special articles, and review articles are also highly recommended for submission to our journal because such articles are often cited, resulting in an increase in the JIF.

Works published in JJR in 2015 included a useful interventional radiology procedure guideline for critical hemorrhage in obstetrics [3], incorporating clinical questions and answers with evidence levels. In addition, a number of review articles have been submitted, two of which were published in JJR in 2015 [4, 5]. Furthermore, we published one letter to the editor [6] that provided constructive comments and suggestions regarding an article published in 2015 in JJR [7].

We will continue to publish special content that introduces recent Japanese Guidelines, to promote review 
articles, and also to encourage valuable discussions among the authors of papers published in our journal. Hopefully, such efforts will attract many readers around the world, thus increasing the number of downloads of JJR articles and the JIF of the journal in the near future.

At the same time, as it is the official journal of the Japan Radiological Society, the aim of this journal has always been the same as that of the society: the promotion of radiology as a science. We continue to publish high-quality research in this field and to promote efforts aimed at improving patient health. We are always happy to accept articles that are relevant to our readership.

We are indebted to the many members and scientists who have contributed to JJR by submitting the best of their work. We also express our sincere gratitude to the reviewers and editorial staff members who dedicate their time and lend their professional expertise to the journal. I am confident that this journal will continue to make good progress in 2016.

\section{References}

1. Tamaki N. The year for our new endeavor: 2015. Jpn J Radiol. 2015;33:1-3.

2. Ehara S. Assessing the scientific and educational value of case reports: an editor's view. Jpn J Radiol. 2011;29:1-2.

3. Sone M, Nakajima Y, Woodhans R, Shioyama Y, Tsurusaki M, Hiraki T, et al. Interventional radiology for critical hemorrhage in obstetrics: Japanese Society of Interventional Radiology (JSIR) procedure guidelines. Jpn J Radiol. 2015;33:233-40.

4. Nakayama T, Ehara S. Spondylolytic spondylolisthesis: various imaging features and natural courses. Jpn J Radiol. 2015;33:3-12.

5. Kitajima $\mathrm{K}$ et al. Present and future role of FDG-PET/CT imaging in the management of head and neck carcinoma. Jpn J Radiol 2015;33 (in press).

6. Wasilewski J, Desperak P. Proximal LAD atherosclerosis: milking-like effect of the septal perforator branches. Jpn J Radiol. 2015;33:523-4.

7. Amanuma M, Kondo T, Arai T, Morita H, Matsutani H, Sekine T, et al. Segmental distribution of calcifications and non-assessable lesions on coronary computed tomographic angiography: evaluation in symptomatic patients. Jpn J Radiol. 2015;33:122-30. 